\title{
Preparação e caracterização microestrutural de cerâmicas ferroelétricas de $\mathrm{Ba}_{0,77} \mathrm{Ca}_{0,23} \mathrm{TiO}_{3}$
}

\section{(Preparation and microstructural characterization of $\mathrm{Ba}_{0.77} \mathrm{Ca}_{0.23} \mathrm{TiO}_{3}$ ferroelectric ceramics)}

\author{
T. Mazon, C. E. B. Silvério, A. C. Hernandes \\ Grupo Crescimento de Cristais e Materiais Cerâmicos \\ Centro Multidisciplinar para o Desenvolvimento de Materiais Cerâmicos \\ Instituto de Física de S. Carlos, Universidade de S. Paulo \\ C.P. 369, 13560-970, S. Carlos, SP \\ tmazon@bol.com.br; hernandes@if.sc.usp.br
}

\begin{abstract}
Resumo
Cerâmicas de titanato de bário e cálcio com composição $\mathrm{Ba}_{0.77} \mathrm{Ca}_{0.23} \mathrm{TiO}_{3}$ (BCT23) foram produzidas usando o método de síntese por reação de estado sólido. Um estudo sistemático de temperatura e tempo de calcinação foi realizado com o objetivo de se determinar as condições experimentais ótimas para a obtenção da fase única BCT23. Observou-se a presença das fases $\mathrm{BaTiO}_{3} \mathrm{e} \mathrm{CaTiO}_{3}$ para os pós calcinados em temperaturas inferiores a $1200^{\circ} \mathrm{C}$ e tempo de até 12 horas. Um aumento na temperatura para $1250^{\circ} \mathrm{C}$ leva a formação da fase BCT23. Os pós calcinados a $1250{ }^{\circ} \mathrm{C}$ foram prensados uniaxialmente em diferentes condições e sinterizados a $1450{ }^{\circ} \mathrm{C} / 4 \mathrm{~h}$. Verificou-se que o método utilizado para a preparação dos compactos influenciou na microestrutura e densidade relativa das cerâmicas. Cerâmicas mais densas foram obtidas a partir da prensagem com pressão uniaxial equivalente a $1 \times 10^{7} \mathrm{~N} / \mathrm{m}^{2}$ dos pós de BCT23 peneirados. Palavras-chave: BCT, perovskitas, síntese de estado sólido.
\end{abstract}

\begin{abstract}
Barium calcium titanate ceramics with composition $\mathrm{Ba}_{0,77} \mathrm{Ca}_{0,23} \mathrm{TiO}_{3}$ (BCT23) were prepared by solid state reaction. A systematic study of temperature and time of calcination was performed aiming to determine the experimental conditions to obtain only BCT23 phase. It was only observed the $\mathrm{BaTiO}_{3}$ and $\mathrm{CaTiO}_{3}$ phases at calcination temperature up to $1200^{\circ} \mathrm{C}$ and time up to $12 \mathrm{~h}$. However, it was possible to obtain the BCT23 phase by increasing the temperature to $1250^{\circ} \mathrm{C}$ for $12 \mathrm{~h}$. The powders calcined at $1250^{\circ} \mathrm{C}$ for $12 \mathrm{~h}$ were uniaxially pressed at different conditions and sintered at $1450^{\circ} \mathrm{C} / 4 \mathrm{~h}$. The method utilized to prepare the compacts influenced on the microstructure and relative density of the ceramics. Denser ceramics were obtained by pressing a BCT23 powder with $1 \times 10^{7} \mathrm{~N} / \mathrm{m}^{2}$.
\end{abstract}

Keywords: $B C T$, perovskite, solid state synthesis

\section{INTRODUÇÃO}

O titanato de bário e cálcio $\left(\mathrm{Ba}_{1-\mathrm{x}} \mathrm{Ca}_{\mathrm{x}} \mathrm{TiO}_{3}-\mathrm{BCT}\right)$ é um material ferroelétrico com estrutura perovskita a temperatura ambiente e está sendo muito pesquisado por apresentar excelentes propriedades físicas. O BCT apresenta temperatura de transição de fase ferro/paraelétrica a $98^{\circ} \mathrm{C}$. A adição de cálcio aumenta o comportamento eletromecânico, os coeficientes eletro-ópticos do material e o intervalo de temperatura de estabilidade da fase tetragonal, evitando uma transição de fase tetragonal-ortorrômbica em temperaturas maiores que $-120^{\circ} \mathrm{C}[1-3]$. Outra vantagem deste material está na possibilidade de preparar capacitores cerâmicos de multicamadas de BCT com eletrodos de baixo custo, tal como níquel, sem perder o bom desempenho dielétrico [4].

Alguns pesquisadores $[5,6]$, no entanto, têm observado que o comportamento da transição de fase ferro/paraelétrica está diretamente relacionado com a preparação da cerâmica BCT. Particularmente, em [6] foi reportado que cerâmicas de BCT com controle da estequiometria dos íons $\mathrm{Ba}^{2+}$ e $\mathrm{Ca}^{2+}$ somente são obtidas a partir do método de co-precipitação dos precursores $(\mathrm{Ba}, \mathrm{Ca}) \mathrm{CO}_{3}$ e $\mathrm{TiO}_{2}$. Segundo eles, a preparação de cerâmicas de $\mathrm{BCT}$ pelo método de síntese convencional do estado sólido é inviável, pois este método não garante uma boa homogeneidade estequiométrica do cálcio. No entanto, os autores não se preocuparam em obter o mesmo tamanho de grãos nos pós de BCT preparados pelos dois métodos. O tamanho de grão obtido na amostra preparada por síntese convencional de estado sólido foi 4 vezes maior que o obtido para o pó preparado pela rota de co-precipitação. Como a não homogeneidade estequiométrica do cálcio é resultante de limitações difusionais, o maior tamanho de grão obtido no pó preparado por síntese do estado sólido pode ter favorecido a obtenção de uma mistura não homogênea. 
Neste trabalho estabelecemos uma rota de processamento para a produção de cerâmicas densas de $\mathrm{Ba}_{0,77} \mathrm{Ca}_{0,23} \mathrm{TiO}_{3}$ (BCT23) com composição estequiométrica, usando o método de síntese por reação do estado sólido, e efetuamos a sua caracterização microestrutural em função dos parâmetros experimentais de processamento.

\section{PROCEDIMENTO EXPERIMENTAL}

O titanato de bário e cálcio foi preparado com a composição $\mathrm{Ba}_{0,77} \mathrm{Ca}_{0,23} \mathrm{TiO}_{3}$ (BCT23), a partir dos precursores $\mathrm{BaCO}_{3}$ (Merck, $99 \%$ de pureza), $\mathrm{CaCO}_{3}$ (Riedel-de-Haën, $99 \%$ de pureza) e $\mathrm{TiO}_{2}$ (Alfa AESAR, 99,9\% de pureza). O fluxograma da preparação é apresentado na Fig. 1.

Quantidades apropriadas dos precursores foram pesadas e moídas por 96 horas em moinho de bolas, usando bolas de $\mathrm{ZrO}_{2}$ (com tamanho $0,94 \mathrm{~mm}$ ) e álcool isopropílico como solvente para o meio de moagem. A moagem teve por finalidade diminuir efetivamente o tamanho das partículas. Em seguida os pós foram calcinados em forno tipo mufla em diferentes condições de temperatura, visando a obtenção da fase única BCT23.

$\mathrm{O}$ pó calcinado a $1250{ }^{\circ} \mathrm{C}$ por $12 \mathrm{~h}$, o qual apresentou apenas a fase BCT, foi novamente moído por $96 \mathrm{~h}$ em álcool isopropílico, prensado uniaxialmente e os compactos obtidos foram sinterizados a $1450{ }^{\circ} \mathrm{C}$ por $3 \mathrm{~h}$. Com a finalidade de obter cerâmicas densas e verificar a influência da pressão utilizada em sua densificação, os compactos foram prensados basicamente em três condições:

(a) Prensagem uniaxial do pó em prensa hidráulica com pressão equivalente a $1 \times 10^{8} \mathrm{~N} / \mathrm{m}^{2}$. (b) Prensagem uniaxial do pó com pressão equivalente a $1 \times 10^{7} \mathrm{~N} / \mathrm{m}^{2}$. (c) Prensagem uniaxial do pó peneirado em peneira com abertura de 100 mesh, com pressão equivalente a $1 \times 10^{7} \mathrm{~N} / \mathrm{m}^{2}$.

Após a moagem da mistura dos precursores, a distribuição do tamanho de partículas e o tamanho médio de partículas foram obtidos por sedigrafia (Micromeritics Sedigraph modelo 5100) e microscopia eletrônica de varredura (MEV) (DSM 960, Zeiss)

As transformações físicas e químicas do pó sem reagir e do pó reagido foram acompanhadas por análise térmica (TG/ ATD) (Netzsch STA 409) no intervalo de temperatura de $25^{\circ} \mathrm{C}$ a $1400{ }^{\circ} \mathrm{C}$, com taxa de aquecimento de $10{ }^{\circ} \mathrm{C} / \mathrm{min}$, com atmosfera de ar sintético e alumina como material de referência. Após a análise por TG/ATD, os pós calcinados em diferentes condições de tempo e temperatura foram caracterizados por difração de raios X (DRX) (Rigaku Rotaflex RU-200B), visando determinar a formação de fases cristalinas.

As densidades dos compactos sinterizados foram determinadas pelo método de Archimedes. A microestrutura das cerâmicas foi examinada por MEV (Zeiss-DSM960) da superfície polida e atacada termicamente.

\section{RESULTADOS E DISCUSSÃO}

As curvas de TG a ATD obtidas para a mistura dos precursores moídos por $96 \mathrm{~h}$ estão apresentadas na Fig. 2. Observamos a presença de uma banda exotérmica até $550^{\circ} \mathrm{C}$, a qual foi atribuída à perda do álcool isopropílico utilizado durante a moagem, correspondendo a uma perda de massa de $0,3 \%$. Nas curvas de ATD é possível observar, na região entre $580^{\circ} \mathrm{C}$ e $750^{\circ} \mathrm{C}$ uma banda exotérmica referente à decomposição do $\mathrm{CaCO}_{3}$ e formação da fase $\mathrm{CaTiO}_{3}$. Os picos endotérmicos a

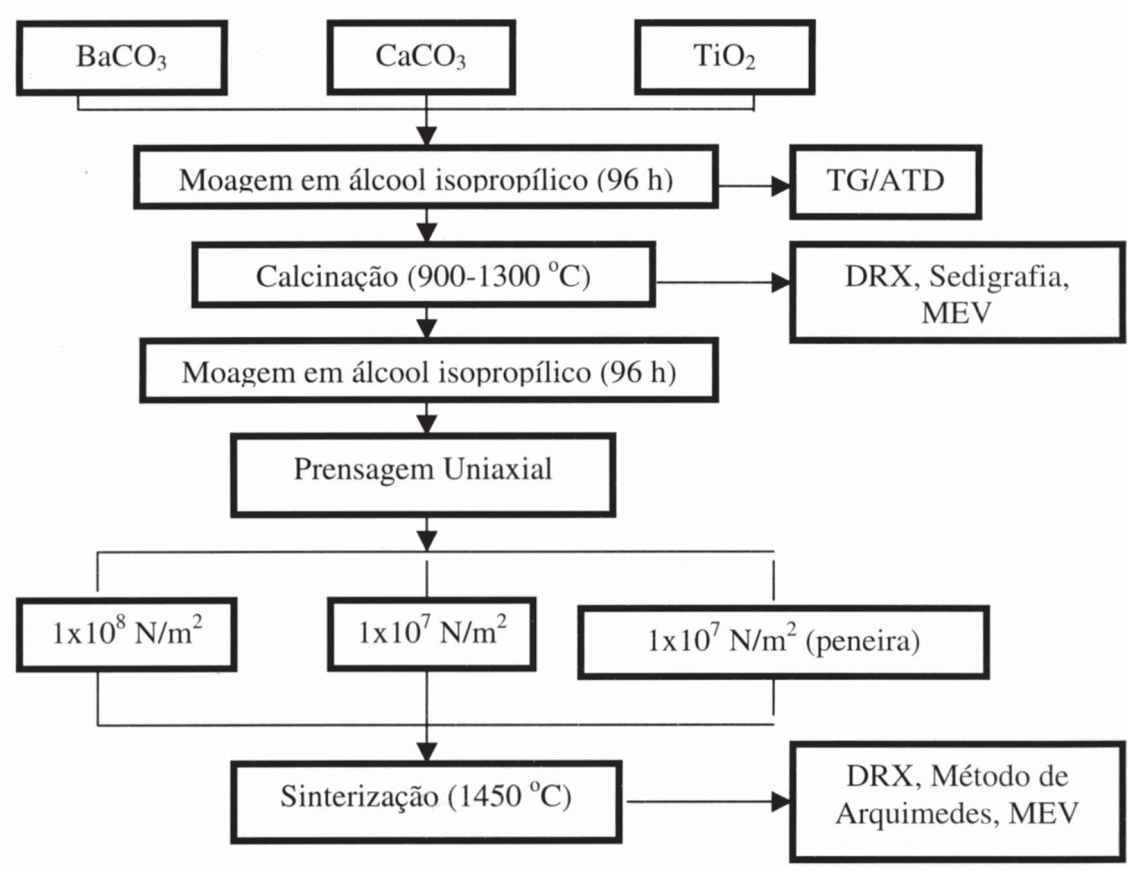

Figura 1: Fluxograma de preparação da cerâmica BCT23.

[Figure 1: Flow chart of BCT23 ceramics preparation.] 


$$
\begin{array}{lll}
\mathrm{CaCO}_{3}+\mathrm{TiO}_{2} & \stackrel{\left(580-750^{\circ} \mathrm{C}\right)}{\longrightarrow} & \mathrm{CaTiO}_{3}+\mathrm{CO}_{2}{ }^{\uparrow} \\
\mathrm{BaCO}_{3}+\mathrm{TiO}_{2} & \stackrel{\left(830-1000^{\circ} \mathrm{C}\right)}{\longrightarrow} & \mathrm{BaTiO}_{3}+\mathrm{CO}_{2}{ }^{\uparrow} \\
0,23\left(\mathrm{CaTiO}_{3}\right)+0,77\left(\mathrm{BaTiO}_{3}\right) \stackrel{\left(1250^{\circ} \mathrm{C}\right)}{\longrightarrow} & \mathrm{Ba}_{0,77} \mathrm{Ca}_{0,23} \mathrm{TiO}_{3}
\end{array}
$$

830 e $990{ }^{\circ} \mathrm{C}$ foram atribuídos à transições estruturais que ocorrem no $\mathrm{BaCO}_{3}[7]$. Estas transições estruturais são acompanhadas de perda de massa, referentes à perda de $\mathrm{CO}_{2}$. $\mathrm{A}$ perda de massa total é de $18 \%$. A $1000^{\circ} \mathrm{C}$ não há mais perda de massa, indicando que ocorreu a formação da fase $\mathrm{BaTiO}_{3}$.

Uma pequena banda exotérmica, sem perda de massa, com o máximo em torno de $1250^{\circ} \mathrm{C}$, também é observada na curva de ATD (Fig. 2). Como não há mais perda de massa nesta temperatura, esta banda foi atribuída à reação entre $\mathrm{CaTiO}_{3} \mathrm{e}$

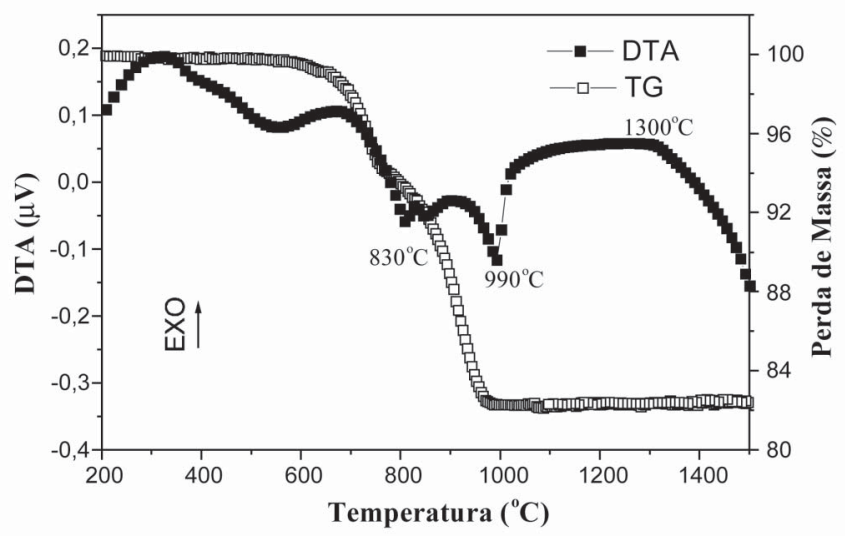

Figura 2: Curvas de TG e ATD da mistura dos pós precursores moída por $96 \mathrm{~h}$; taxa de aquecimento $10^{\circ} \mathrm{C} / \mathrm{min}$, atmosfera de ar sintético. [Figure 2: DTA and TG curves for non-reacted powders milled for $96 \mathrm{~h}$. Heating rate of $10^{\circ} \mathrm{C} / \mathrm{min}$ and air.] (a)

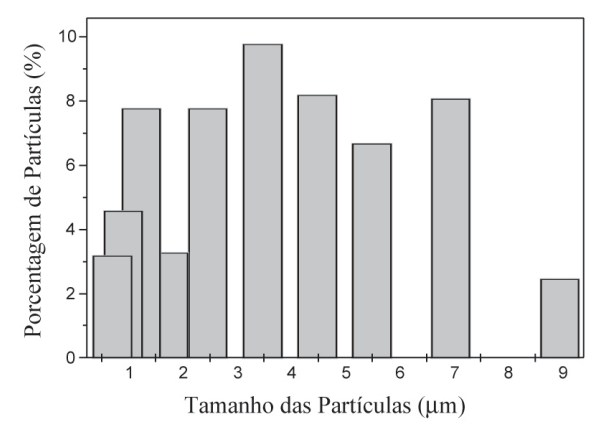

(b)

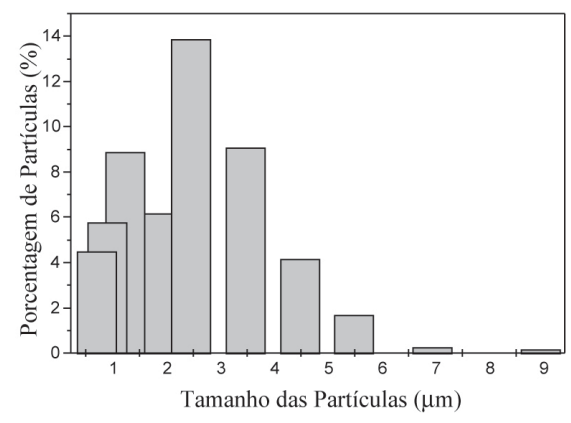

(c)

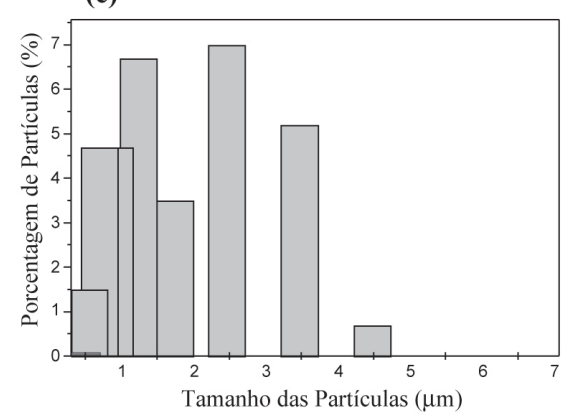

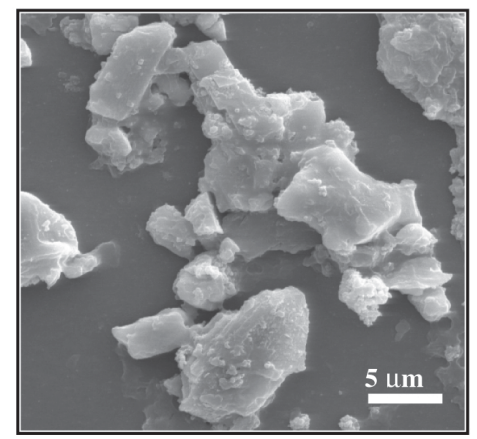
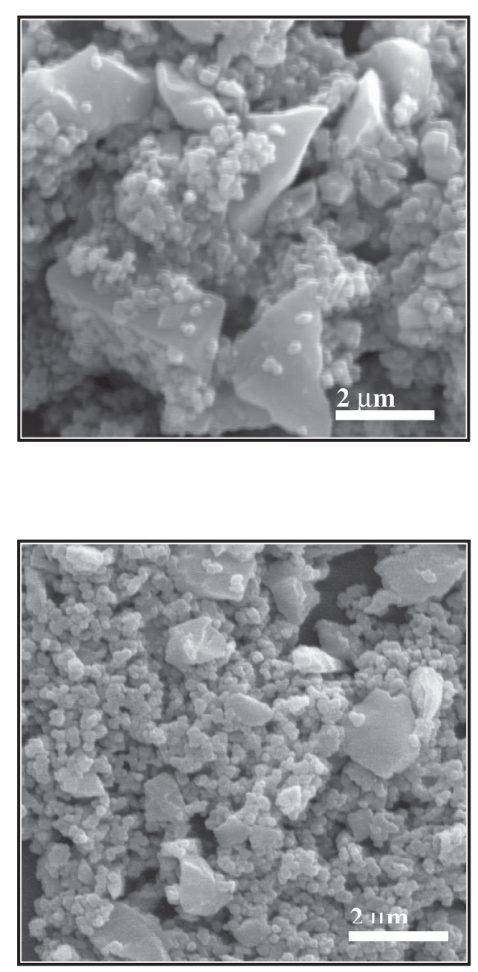

Figura 3: Distribuição do tamanho de partícula e sua morfologia para diferentes condições de tempo de moagem: (a) 24 h; (b) 72 h; (c) 96 h. [Figure 3: Sedigraph results and SEM micrographs of precursor powders milled at different time conditions: (a) $24 h$; (b) $72 h$; (c) 96 h.] 


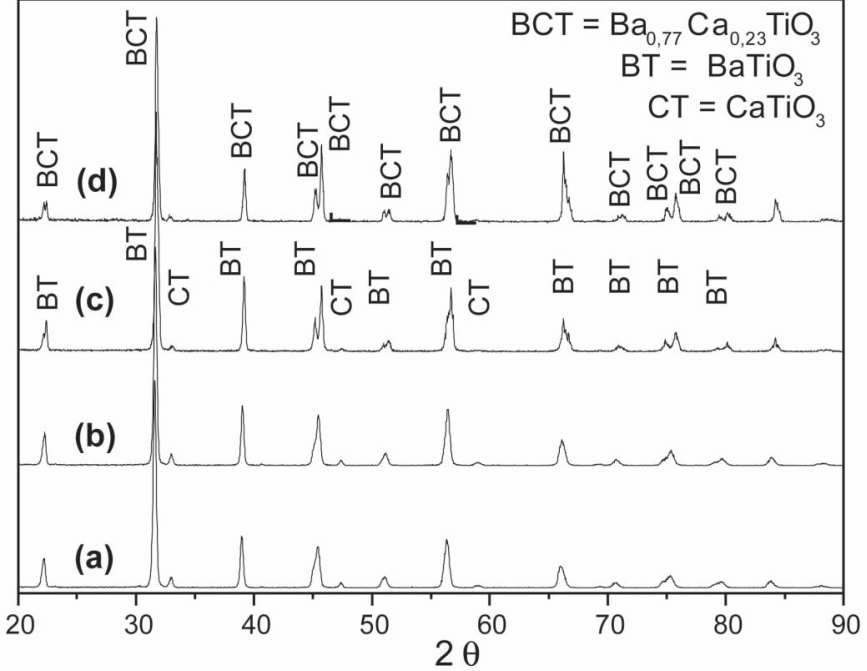

Figura 4: Difratogramas de raios $\mathrm{X}$ dos pós de $\mathrm{BCT}$ calcinados sob diferentes condições: (a) $1100^{\circ} \mathrm{C} / 6 \mathrm{~h}$; (b) $1200^{\circ} \mathrm{C} / 6 \mathrm{~h}$; (c) $1250^{\circ} \mathrm{C} / 6 \mathrm{~h}$; (d) $1250{ }^{\circ} \mathrm{C} / 12 \mathrm{~h}$.

[Figure 4: XRD patterns of the calcined powders at different temperature conditions: (a) $1100{ }^{\circ} \mathrm{C} / 6 \mathrm{~h}$; (b) $1200^{\circ} \mathrm{C} / 6 \mathrm{~h}$; (c) $1250{ }^{\circ} \mathrm{C} / 6 \mathrm{~h}$; (d) $\left.1250^{\circ} \mathrm{C} / 12 \mathrm{~h}.\right]$

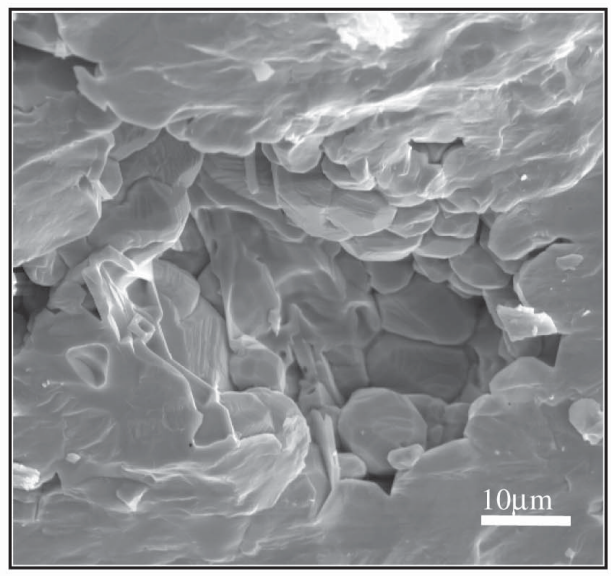

(a) $74 \%$

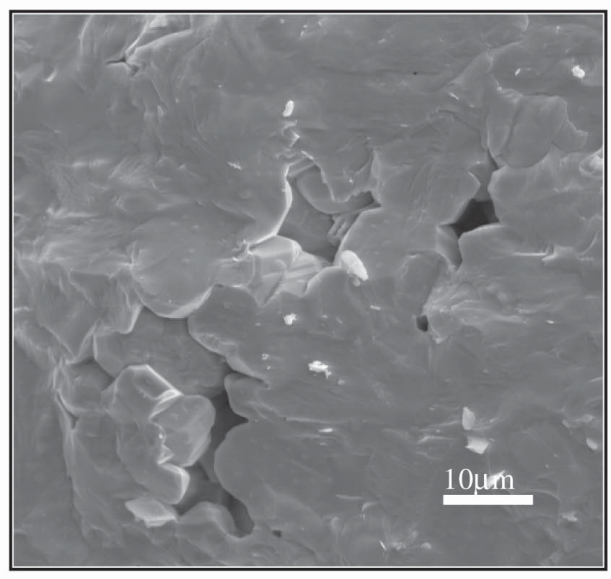

(c) $98 \%$
Tabela I - Densidades relativas das cerâmicas de BCT23 prensadas sob diferentes condições e sinterizadas a $1450^{\circ} \mathrm{C}$ por $4 \mathrm{~h}$.

[Table I - Relative densities of the BCT23 ceramics pressed under different conditions and sintered at $1450{ }^{\circ} \mathrm{C} / 4 \mathrm{~h}$.]

Forma de prensagem:

Densidade

Relativa (\%)

\begin{tabular}{ll}
\hline Pó prensado com pressão de $1 \times 10^{8} \mathrm{~N} / \mathrm{m}^{2}$. & 74 \\
\hline Pó prensado com pressão de $1 \times 10^{7} \mathrm{~N} / \mathrm{m}^{2}$. & 90 \\
\hline Pó peneirado e prensado com pressão de $1 \times 10^{7} \mathrm{~N} / \mathrm{m}^{2}$. & 98 \\
\hline
\end{tabular}

$\mathrm{BaTiO}_{3}$ para formação da fase BCT. Desta forma, o processo para a formação da fase BCT pelo método utilizado pode ser descrito pelas seguintes reações:

A distribuição do tamanho de partículas e os valores de tamanho médio de partículas foram obtidos por sedigrafia e MEV para as amostras moídas por tempos diferentes. Os resultados estão apresentados na Fig. 3. Podemos notar que o tamanho médio das partículas diminui com o aumento do tempo de moagem e o intervalo de distribuição do tamanho

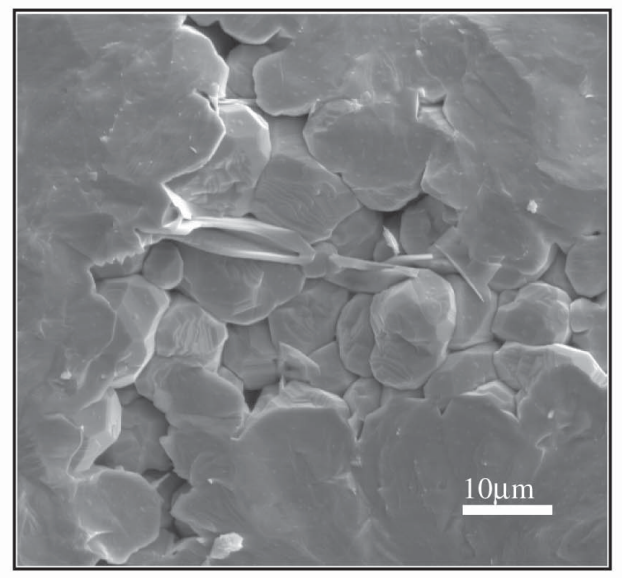

(b) $90 \%$

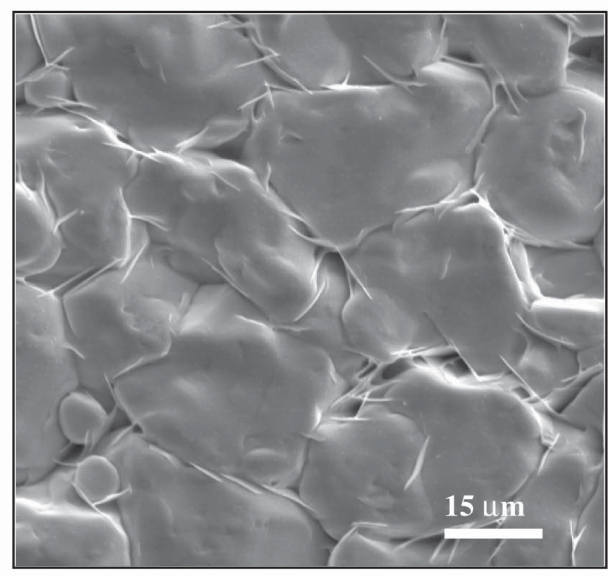

(d) $98 \%$

Figura 5: Micrografias obtidas por microscopia eletrônica de varredura das cerâmicas de BCT sinterizadas a $1450{ }^{\circ} \mathrm{C}$ por 4 h. [Figure 5: SEM micrographs of specimens sintered at $1450^{\circ} \mathrm{C}$ for $4 \mathrm{~h}$.] 
de partículas se torna mais estreito. Comparando o tamanho médio de partículas obtidos por sedigrafia e por MEV, observamos que há uma pequena diferença nos valores para as amostras moídas por um tempo maior que $72 \mathrm{~h}$. O tamanho médio de partículas obtido por MEV, para a amostra moída por $96 \mathrm{~h}$ está em torno de $0,4 \mu \mathrm{m}$, enquanto que o valor obtido por sedigrafia é de $1,5 \mu \mathrm{m}$. Esta diferença está relacionada à aglomeração das partículas durante a análise de sedigrafia.

Tamanho de partícula pequeno é um fator fundamental para obter maior reatividade entre os compostos óxidos. Principalmente no caso do BCT, onde composições com mais de $20 \mathrm{~mol} \%$ de $\mathrm{Ca}^{2+}$ precisam de um tempo maior de moagem para garantir que todo $\mathrm{Ca}^{2+}$ se distribua de uma maneira homogênea, para favorecer a formação da fase BCT durante a calcinação. $\mathrm{O}$ tempo de moagem otimizado neste trabalho foi de 96 h, garantindo a obtenção de fase única de BCT23 e uma estreita distribuição do tamanho de partículas. Nesta condição, o tamanho médio de partículas foi da ordem do obtido por [7] utilizando uma rota semi-úmida para preparar pós de BCT.

Os difratogramas de raios $\mathrm{X}$ obtidos para os pós de $\mathrm{Ba}_{0,77} \mathrm{Ca}_{0,23} \mathrm{TiO}_{3}$ calcinados em diferentes condições de tempo e temperatura estão apresentados na Fig. 4. Observa-se a presença das fases BT e CT para temperaturas de calcinação inferiores a $1250{ }^{\circ} \mathrm{C}$. Um aumento na temperatura e no tempo de calcinação para $1250^{\circ} \mathrm{C}$ por $12 \mathrm{~h}$ favoreceu a formação da fase BCT23. A temperatura de calcinação para a obtenção da fase BCT23 neste trabalho foi $100{ }^{\circ} \mathrm{C}$ menor que a citada na literatura [8], quando se utiliza a mesma composição de cálcio. O menor tamanho médio de partículas obtido em nosso processo possibilitou obter resultado semelhante, mas em temperatura mais baixa.

Visando determinar a melhor metodologia de prensagem para obter cerâmicas densas de $\mathrm{BCT}$, o pó calcinado a $1250^{\circ} \mathrm{C}$ por $12 \mathrm{~h}$ foi prensado em diferentes condições e sinterizado a $1450{ }^{\circ} \mathrm{C}$ por $4 \mathrm{~h}$. Os resultados de densidades relativas obtidas para os compactos sinterizados estão apresentados na Tabela I e as micrografias obtidas por microscopia eletrônica de varredura para as cerâmicas estão apresentadas na Fig. 4.

A forma de prensagem influenciou diretamente na densidade relativa da cerâmica. A maior densidade relativa foi obtida para o pó peneirado e prensado sob uma pressão uniaxial de $1 \times 10^{7} \mathrm{~N} / \mathrm{m}^{2}$. A utilização de pressões maiores durante a compactação dos pós leva à formação de defeitos nos compactos, dificultando a densificação dos corpos cerâmicos.

Embora as cerâmicas de BCT apresentassem 98\% de densidade relativa, pode-se notar que ocorreu um crescimento exagerado dos grãos e a presença de uma fase tipo vítrea segregada no contorno de grãos (Fig. 4d). Análises por EDX apontam que esta fase é rica em titânio. O crescimento exagerado de grãos e segregação de Ti no contornos de grãos também foram verificados em cerâmicas de $\mathrm{BaTiO}_{3}[10]$. Esta fase segregada nos contornos de grãos pode ser prejudicial para as propriedades elétricas do material. Novos trabalhos estão sendo realizados visando a eliminação desta fase.

\section{CONCLUSÕES}

Desenvolvemos uma metodologia para a obtenção de cerâmicas densas de BCT23 com alta reprodutibilidade no processo. As condições experimentais otimizadas para a calcinação foram $1250{ }^{\circ} \mathrm{C}$ por $12 \mathrm{~h}$ em forno de atmosfera aberta. A rota de preparação dos pós de $\mathrm{BCT}$ estabelecida neste trabalho favoreceu a obtenção da fase BCT23 em temperaturas mais baixas que as citadas na literatura. O método de prensagem influenciou na microestrutura e densificação das cerâmicas. Cerâmicas com $98 \%$ de densidade relativa foram obtidas a partir do pó peneirado em peneira com abertura de 100 mesh e prensado uniaxialmente com pressão equivalente a $1 \times 10^{7} \mathrm{~N} / \mathrm{m}^{2}$.

\section{AGRADECIMENTOS}

Os autores agradecem a FAPESP e ao CNPq pelo apoio financeiro.

\section{REFERÊNCIAS}

[1] A. Mazur, C. Veber, O. F. Scirmer, C. Kuper, H. Hesse, Radiat. Eff. Deffect. Sol. 150 (1999) 281.

[2] H. Veenhuis, T. Boger, K. Peithmann, M. Flaspohler, K. Buse, R. Pankrath, H. Hesse, E. Kratzig, Appl. Phys. B. 70 (2000) 797.

[3] C. H. Kuper, R. Pankrath, H. Hesse, Appl. Phys. A. 65 (1997) 301.

[4] J. M. Hebert, Ceramic Dielectrics and Capacitors, Gordon and Breach, New York, London (1985) cap. 6.

[5] V. S. Tiwari, D. Pandey, P. Groves, J. Phys. D 22 (1989) 837. [6] D. Pandey, V. S. Tiwari, T. B. Singh, L. Pandey, O. Parkash, P. R. Rao, Phase Trans. 9 (1987) 11.

[7] V. S. Tiwari, N. Singh, D. Pandey, J. Am. Ceram. Soc. 77 (1994) 1813.

[8] R. C. De Vries, R. Roy, J. Am. Ceram. Soc. 38 (1955) 142.

[9] W. Z. Black, J. G. Hartley, Thermodynamics SI version, Harper Collins, New York (1991) 675.

[10] Y. Yamamoto, Brit. Ceram. Trans. 94, 5 (1995) 196.

(Rec. 18/07/03, Ac. 20/02/04) 